Fecha de entrega: 18 de noviembre de 2009

Fecha de aprobación: 8 de marzo de 2010

\title{
EL SOCIALISMO RAIZAL DE FALS BORDA
}

\section{FALS BORDAS'S RAIZAL SOCIALISM}

\author{
Diego Jaramillo Salgado*
}

\section{Resumen}

El ensayo explora los principios básicos y los supuestos teóricos del socialismo raizal propuesto por el sociólogo colombiano Orlando Fals Borda. Este socialismo es presentado como una propuesta enraizada en la Investigación Acción Participativa (IAP), haciendo énfasis en su origen en las experiencias históricas revolucionarias de los pueblos originarios y diversos sectores populares de América Latina. Igualmente, se resalta su contenido popular, humanista, democrático y epistémico. El ensayo también explora sus acercamientos y sus diferencias con el pensamiento de Marx.

\section{Palabras clave}

Socialismo, raizal, cultura, popular, epistemología, revoluciones.

\begin{abstract}
The essay explores the basic principles and the theoretical assumptions of the rooted socialism proposed by the Colombian sociologist Orlando Fals Borda. This socialism is shown as a deep-rooted proposal on the Participative Action Research (PAR), setting emphasize in its origin on the revolutionary historical experiences of the native and diverse popular areas of Latin-America. Likewise, its popular, humanist, democratic and epistemic contents are highlighted. The essay also explores its encounters and its differences to the thoughts of Karl Marx.
\end{abstract}

Doctor en Estudios Latinoamericanos. UNAM. Profesor jubilado de la Universidad del Cauca. Correo electrónico:djara9@hotmail.com 


\section{Key words}

Socialism, rooted, culture, popular, epistemology, revolutions.

\section{Introducción}

Cuando Fals Borda recibió la Orden Gerardo Molina, en septiembre de 2002, refiriéndose al maestro que le daba su nombre, planteó la "dualidad entre el intelectual y el político" como "problemática", en relación con el devenir de la universidad colombiana, sujeto a vaivenes "cíclicos" de énfasis en la acción, en unos períodos, y de aislamiento de la realidad social, al convertirse en tecnócratas y apolíticos, en otros. En ese momento anunciaba la llegada de un ciclo, que superaría o sintetizaría los anteriores, expresado en la figura del "equilibrio vivencial que tenía el Maestro Molina entre el intelectual y el político" (Fals Borda, 2003, p. 139). Orientación que se pondría en dirección del largo proceso acreditado por él a través de la adopción de la Investigación Acción Participativa (IAP); pues en ella se produce la confluencia de una teoría y metodología académicas con el movimiento práctico de las comunidades y de los pueblos. Si bien la investigación en este caso no obedece a un compromiso político directo con una organización política, como es la crítica que subyace al carácter problemático de esa dualidad, si lo es desde el punto de vista de los fines implícitos en ese tipo de búsquedas.

De la misma manera, su propuesta de un socialismo partió del estudio y recorrido de nuestra propia historia, basándose en la historiografía de fuentes primarias y secundarias, y en la respuesta a demandas políticas que planteaban la sociedad y los movimientos políticos en que militaba. Aunque, a decir verdad, él mismo hace una autocrítica a su formación inicial de "sociólogo positivista" que le "impidió entender a las utopías como algo digno de consideración académica" (Fals
Borda, 1992, p. 1). Es decir, inmerso en la lógica y en los procesos de los desarrollos de las ciencias, por fuera de su articulación con la vida social y cultural. A renglón seguido da cuenta de que fueron la "experiencia extrauniversitaria" y el "testimonio de los anhelos revolucionarios de la década siguiente (los tórridos años 60)" los que cambiaron su visión y lo aproximaron a las utopías.

De ahí, que los principios del socialismo por él estructurados no se circunscriben al estudio minucioso de los clásicos del socialismo; también se desprenden del movimiento práctico de los pueblos. Mucho más en un caso como el colombiano en que la investigación en el campo y en diferentes regiones, en las décadas del setenta y el ochenta del siglo pasado, obligatoriamente imponían el encuentro del investigador con actores del conflicto armado. En particular de las guerrillas que planteaban estrategias de construcción del socialismo, y su inserción en la dinámica de los movimientos sociales alentaban la lucha por una nueva sociedad en esa dirección. No propiamente desde una estrategia educativa centrada en discursos que clarificaran su significado. Más bien, desde la lucha directa, en este caso armada y a través de la movilización, como pedagogía hacia la nueva formación social.

\section{De los principios y supuestos teóricos del socialismo}

Quizá eso nos permita identificar, en los principios y supuestos teóricos por él propuestos, una raigambre en las luchas propias de los pueblos, las subversiones producidas a lo largo de la historia, las revoluciones en la transición del feudalismo al capitalismo, sus 
diferentes modalidades en América Latina, y las dinámicas de las comunidades y de los pueblos reaccionando contra los intentos de arrasamiento y hegemonización.

No parte entonces de principios marxistas, que ya eran socializados en Colombia cuando regresó al país en 1948. Más bien tiene como punto de partida las teorías del conflicto y del cambio social, y el análisis de la crítica conflictividad que, bajo la forma de violencia, se radicalizó en Colombia a partir de ese año con el asesinato de Jorge Eliécer Gaitán. Registro en el que participó, conjuntamente con el sacerdote Germán Guzmán y Eduardo Umaña Luna, por medio de la investigación que condujo a la elaboración del texto clásico La Violencia en Colombia, publicado en 1962. Pues no solamente confirmaba una especie de reacomodamiento de la que luego denominara "Primera República", sino también la constatación de las dificultades de las masas para poder lograr una transformación social. Al mismo tiempo, las limitaciones que tuvieron partidos y grupos como el comunista, y los socialistas para dirigir los procesos de transformación social. A pesar de ello consideraba inevitable encontrar una sedimentación de prácticas comunitarias, relegadas u olvidadas que encerraban en su interior el germen de un nuevo país. Base esencial de lo que serían los fundamentos básicos del socialismo que consideró pertinentes para el país y para América Latina.

Ya desde su texto Las Revoluciones inconclusas en América Latina, escrito en 1968, insinuaba esta directriz; pues estaba orientado a encontrar los hitos de revoluciones que no llegaron a ser, pero dejaron signos que, actualizándose, reemprendieron otros procesos explicativos del fermento revolucionario que recorría a la región en la década del sesenta del siglo pasado. Orientación por sí renovadora, pues se salía de la discusión de la izquierda del período que circunscribía la lucha por el socialismo a la adscripción que se hiciera a una de las corrientes del marxismo internacional. Luego, en El Socialismo que queremos, escrito en 1982, lo hace explícito al afirmar:

El socialismo nuestro, bien entendido, ha sido y seguirá siendo una búsqueda creadora de vías propias para resolver a favor de los intereses generales y del pueblo trabajador, con equidad y autonomía, los problemas que nos plantea la historia de nuestros orígenes, el mundo en que vivimos, y los imperialismos capitalistas que nos asedian (Fals Borda, 1982, p. 1).

Metodología y principios que no lo abandonarán jamás y serán parámetros que le posibilitarán el desarrollo de su capacidad creativa. Pues no solamente se trataba del seguimiento de los desarrollos teóricos que se producían en el momento, sino fundamentalmente el descubrimiento de los aspectos de la propia historia cultural y de resistencia y de lucha que pueden ser terreno fértil para la nueva sociedad que se quiera construir.

Por ello, un punto de partida para identificar los supuestos que le dan sentido al socialismo que propone es su identificación de cuatro órdenes sociales en la historia nacional. El orden social lo define como "El conjunto de formas de vida actuante que se manifiestan en una sociedad durante un período histórico, a través de mecanismos conformados por elementos socioculturales" (Fals Borda, 2008, p. 36). Necesarios en su estudio porque logra mostrar las diferentes tradiciones e identidades que están ancladas en la historia cultural del país, y los períodos o acciones de subversión que enriquecieron la memoria histórica, germen ineludible de cualquier nueva construcción social.

Un primer orden es el ayllico que da cuenta de aquél construido por el aborigen pre- 
colombino. En el cual encontrará rasgos específicos que darán sentido a la formación de la nacionalidad. El segundo, es "el señorial y de castas, impuesto por los invasores españoles en el siglo XVI", el tercero es el "burgués-conservador ubicado a partir del siglo XIX". El cuarto es el "social burgués que se desarrolla a lo largo del siglo XX". Un quinto orden sería la opción de construcción de una sociedad que superaría todos los órdenes superiores, pero integrándolos en aquellos aspectos que sean positivos para el desarrollo humano (Fals Borda, 2008, p. 10).

A su vez, estos órdenes serían transformados por sucesivas “subversiones morales". Qué entiende, entonces, por subversión? Es “aquella condición que refleja las incongruencias internas de un orden social descubiertas por miembros de éste en un período histórico determinado, a la luz de nuevas metas valoradas que una sociedad quiere alcanzar" (Fals Borda, 2008, p. 32). Definición a la que llega después de analizar el significado negativo y peyorativo que generalmente se le da y encontrarle su opción positiva en los procesos de transformación social y el contenido moral que le es congruente. La primera subversión moral es:

La cristiana, representada por la cruz y la espada de los europeos. La segunda es la liberal inspirada en la Ilustración de mediados del siglo XIX que tuvo también visos socialistas y radicales, valores frustrados por el orden burgués. La tercera es la socialista de comienzos del siglo $\mathrm{XX}$ a su vez seguida por la alianza del orden social burgués (Fals Borda, 2008, p. 10).

Consecuentemente, admitirá una cuarta subversión moral que denomina la neosocialista que anunciaría el quinto orden propuesto.
De esta manera, la opción del socialismo está inscrita en los sentidos culturales, políticos y sociales que los pueblos han construido a través de la historia. No se trataría, entonces, de la aplicación de formatos doctrinarios a una sociedad que no los reconoce como propios. Tampoco daría cabida a la construcción que hicieran partidos u organizaciones pretendiendo imponer a la sociedad modelos experimentados en otros países. No quiere decir esto que excluya opciones teóricas y doctrinarias o experiencias políticas producidas en otras sociedades. Se trata más bien de apropiarse de ellas, pero en función de lo que vaya marcando la propia historia nacional y lucha de los pueblos. Porque, según él "es tiempo, pues, de retomar nuestra historia y geografía real, apreciar más nuestras culturas y revivir los valores fundantes de nuestras naciones y comunidades" (Fals Borda, 2007, p. 17).

Por ello enfatiza que su propuesta debe partir de identificar los contenidos de la vida cultural y comunitaria de los que denomina pueblos originarios, que asume como "fundantes de nuestra nacionalidad". En cuanto hay unas raíces culturales y sociales en la sociedad $\mathrm{y}$ en la historia nacional que son insumo básico para cualquier nueva sociedad que se quiera construir. A partir de allí se estructuran prácticas culturales y valores que se vuelven necesarios, siempre y cuando se produzca su reconocimiento, para la transformación social. ¿Cuáles son estos pueblos originarios para Fals Borda?:

Hemos destacado cuatro: los indígenas primarios, los negros de los palenques, los negros cimarrones rebeldes contra los esclavistas desde el siglo XVII; los campesinos-artesanos pobres antiseñoriales de origen hispánico de la época colonial, y los colonos y patriarcas del interior agrícola en expansión desde el siglo XIX (Fals Borda, 2007, p. 16). 
Dando pie, de este modo, a establecer relaciones con procesos similares en América Latina que, sin repetirse, posibilitan la opción de dar forma a un socialismo como el que propone para Colombia. Fundamentalmente por historias y procesos similares que la memoria cultural permitirá deshilvanar en la formación de una nueva sociedad.

Delimita, en esta forma, el campo de acción de unos actores que cimentan las raíces de la cultura nacional y portan valores que trascienden los modos de producción en que se originaron para hacerse necesarios de reivindicar en una nueva construcción social. ¿Desconoce esta interpretación la existencia de otros actores sociales en la vida nacional? De ninguna manera, más bien enfatiza los puntos de arranque que podrían darle identidad a la nación al confrontar y superar todas aquellas formas de hegemonía y dominación que las sometieron y relegaron haciendo de la exclusión, de la invisibilidad, o de su destrucción el signo permanente de afirmación de prácticas y valores producidos allende nuestras fronteras. Ya lo planteaba, incluso, en su estudio sobre cooperativas de Colombia, Ecuador y Venezuela, por allá a finales de la década del sesenta del siglo pasado, al proponer un "modelo de acción cooperativa":

adecuado a las características históricas y a la idiosincrasia del campesinado raso, un modelo basado en los aspectos dinámicos de su tradición y suficientemente autónomo y decidido como para liberar a los campesinos menos afortunados de las condiciones de opresión en que viven (Fals Borda, 1972, p. 18).

Conclusión a la cual llegaba después de verificar las formas de asimilación, por parte de las políticas dominantes, del sistema cooperativo que se desarrollaba en América
Latina. Sobre todo, si se tiene en cuenta que no pocos le apostaban a que su generalización fuera una salida a los impactos de capitalismo. Tendencia en la que se inscribe el autor, pero reinscrita en una sociedad socialista y democrática.

El socialismo adquiere así un engranaje que construyen, o aportan a su realización, los pueblos y las comunidades. Al mismo tiempo, se articula con procesos que la historia nacional fue registrando como expresiones de un socialismo que provenía de Europa, pero que en las luchas políticas internas fue estableciendo sus propios códigos culturales. Además, la vida de las comunidades, y fragmentos de la propia historia nacional, expresan innumerables valores y prácticas culturales que muy bien pueden inscribirse dentro de procesos que se orienten hacia el socialismo. No circunscritas a una clase social, el proletariado, ni a la lucha de clases; mucho menos a dar solidez a una sola perspectiva en función de la dictadura proletaria, como lo establecían, diferentes organizaciones marxistas.

Por eso para él tiene un significativo valor la revolución de mitad del siglo XIX, jalonada por las sociedades democráticas y por el liberalismo radical que le dieron al país una primera experiencia con esa denominación; así los supuestos y principios políticos fueran los del liberalismo. El golpe de estado del general José María Melo y su corto período de gobierno trazarían un derrotero al que la historia nacional recurriría permanentemente. En especial, como el camino que pudo seguir la república para implementar los logros que la humanidad había efectuado en sociedades europeas y en Norte América. La frustración de esa acción por el socialismo quiso ser superada bajo una nueva orientación en el siglo XX, a partir de la creación del Partido Socialista en 1919 y del Partido Socialista Revolucionario en 1926. Si bien, Fals Borda no hace mucho énfasis en el carácter mar- 
xista de las organizaciones que siguieron esa orientación desde el denominado Grupo Comunista creado por Luis Tejada, y aún desde la corriente que en su nombre se expresó en el partido que le antecedió, le es inevitable admitir que allí se estructuró un proceso de una nueva subversión moral. Porque no se hacía sólo en nombre de reformar las instituciones a través de la obtención de derechos conculcados a los trabajadores y a las masas populares, sino también, porque llevaban en sus prácticas la lucha por la construcción de una nueva sociedad, identificada como socialista.

\section{Socialismo raizal: hacia una definición}

¿Qué es, entonces, lo que Fals Borda define propiamente como socialismo raizal? Quizá no sea necesario hacer un seguimiento a su denominación como tal, pues los contenidos de su concepto lo preceden en su definición a lo largo de toda su trayectoria investigativa y de su actividad política. Sin embargo, sirva como referencia que en su literatura aparece predominantemente de manera explícita a partir de 2003. Antes de ello había recurrido a un término de los huitotos "Kaziyadú", "que significa despertar, renacer, amanecer, y también es la traducción al Huitoto de la palabra "desarrollo" (Fals Borda, 2001, p. 12). Cuyo significado lo orienta hacia el desarrollo alternativo, en el que se incluyen todos los principios y estrategias del discurso socialista estructurados desde la década del setenta. Lo cual podría significar que la lengua de un grupo étnico le daba un instrumento lingüístico para reafirmar los postulados del proyecto de sociedad que había construido.

Habría que empezar por registrar su asociación u homologación con el humanista, el Ecosocialismo, la Democracia radical o la Democracia participativa. No en vano, pues el carácter humanista estaría dado en la reivindicación de los derechos que, como los llamados derechos humanos, asume como consustanciales a todo ser humano o son fuente de su dignificación como tal. Es decir, considera inevitable, para una sociedad que pretenda superar las anteriores que fueron inferiores a la realización humana, reivindicar para sí aquello que haga posible una mejor convivencia social, no solamente de los pobladores de la sociedad que subvierte el orden establecido sino de todas aquellas que coexisten en el universo. De igual manera, las amenazas que se ciernen sobre la humanidad por el desarrollo extensivo de la industria, sin ningún control. La tala indiscriminada de los bosques, los ejercicios nucleares y el uso de armas químicas y biológicas y de las llamadas regulares, crean una amenaza a la sobrevivencia humana que un proyecto socialista no puede ignorar, por lo cual debe ser la base de sus principios para la nueva realización social.

Asimismo, el autoritarismo, la jerarquización verticalizada en el ejercicio de la política, la anulación o limitación de las libertades; tanto en países capitalistas como en los que vivieron y viven las experiencias socialistas, hacen de la democracia radical un parámetro insustituible en el ejercicio de la vida socialista. Mucho más, si la memoria histórica da como elemento necesario el reconocimiento de que el ejercicio del poder no puede seguir dándose desde núcleos burocráticos que desconocen e ignoran al conjunto de la sociedad para la cual gobiernan. En el caso de Fals Borda, se vuelve apremiante que esta perspectiva tenga en cuenta las dinámicas regionales y territoriales. No sólo a través de un proceso de descentralización producido desde arriba, sino también a partir del reconocimiento de la diversidad étnica y cultural y de la participación directa de sus pobladores en las políticas que rigen su destino.

El socialismo raizal integra los anteriores aspectos como elementos constitutivos de 
su propia definición. Es decir, que un ejercicio ecológico, una democracia radical y una estrategia ecologista son condiciones necesarias para pensar en este nuevo tipo de socialismo. Tan articulado lo están, que justamente la reivindicación de lo humano no se hace solamente por el humanismo que la modernidad creó. Lo es también porque los pueblos y las comunidades han dado muestras de dignificación de la vida. Sus prácticas enseñan una persistente lucha por las libertades, la autonomía, y el reconocimiento; sin que necesariamente tengan la formación escolar que los haya predispuesto en esa dirección. Fals Borda reivindica la lucha de las sociedades por garantizar un equilibrio ecológico entre el hombre y la naturaleza que van más allá del lucro y de la rentabilidad. En particular hace un gran reconocimiento de lo que esto significa en comunidades indígenas y afrocolombianas; resaltando sus múltiples enseñanzas en diferentes regiones del país y de América Latina.

De igual manera, reitera permanentemente las experiencias que sobre el ejercicio de la democracia radical se han dado en el país. Una lejana, la del liberalismo radical de mitad del siglo XIX, considerada por algunos como un tipo de socialismo, (Vargas, 1972; Jaramillo, 1997) que se concreta en el ejercicio del poder del Estado por parte del general José María Melo y tiene su concreción normativa en la Constitución de 1863. Otra, no exenta de críticas, es la de la "Revolución en Marcha", 1936, en que fueron puestos a prueba principios básicos de la democracia liberal. Muchos otros, son registrados en el país a través de asambleas constituyentes o de formaciones de región, como en el reconocimiento que le hace a los intentos de formación de la Región Surcolombiana en el periodo 2001-2003. En su interregno destaca la importancia que tuvo la Asamblea Constituyente del 91 y su producto, la actual Constitución Nacional.
El socialismo raizal lo define como aquél que "toma en cuenta las raíces histórico-culturales y de ambiente natural de nuestros pueblos de base" (Fals Borda, 2007, p. 15). Planteado de esta manera, este socialismo parte de una reconstrucción de la simbología, los mitos, las ritualidades, el lenguaje y las prácticas y procesos culturales que las comunidades y los pueblos han construido a través de la historia. Significa esto que, más allá de las contradicciones de clase y de su correlación con los cambios que se han producido en los modos de producción, hay unas prácticas humanas, y prácticas culturales que se vuelven universales; así en su origen hayan partido de una experiencia específica y particular. No se trata de desconocer o ignorar que hay sociedades con confrontaciones de clase. Es más bien el reconocimiento de que, para la superación de la lucha de clases, es conveniente incorporar, en un proyecto político y social, principios y valores de prácticas culturales que han permanecido a través de los cambios. A la vez, que no hay solamente lucha de clases entre dos polos opuestos de la sociedad; hay múltiples expresiones de la conflictividad social que hacen la historia y que no pueden desconocerse en un proceso de construcción de una nueva sociedad.

En esta dirección, no cabría la identificación de clases sociales protagónicas o vanguardias en la acción de transformación. El papel o la jerarquía que uno $u$ otras pudieran tener en el proceso, no se establecen por una condición a priori al que deben someterse las luchas sociales o las formas organizativas que se vayan estructurando. Es la dinámica de la movilización social de los movimientos y organizaciones sociales, y de las organizaciones políticas que actúan en correspondencia con ello, las que definirán el rol que cada clase o sector social puede tener en el proceso de transformación. Sin embargo, si es claro que a un orden burgués no se le puede encontrar un proceso de transformación que esté ali- 
mentado y liderado por quienes ejercen su control y su dominio. Sin enfatizar en la lucha de clases, hay un implícito reconocimiento de ella al aceptar que un nuevo orden sólo se produce si se estructura de abajo hacia arriba. Es decir, si el nuevo poder logra constituirse a partir de los que fueron sometidos y explotados, subyugados y vilipendiados, en el orden económico y social que se quiere superar. No obstante, no es claro que el socialismo por él propuesto prescinda de clases sociales que existan porque lo hacen a través de la plusvalía, de la ganancia o de la renta de la tierra. Sólo que ellas garantizarían su existencia en cuanto se supediten a los fines colectivos que determine el nuevo orden social.

No estamos entonces frente a un postulado marxista que socialice totalmente los medios de producción. Estaríamos más bien identificando una especie de socialdemocracia, pero con las limitantes que le impondría el socialismo raizal en relación con los impactos ecológicos de la industria y de las limitaciones que el estado establecería para que fuera efectiva una redistribución del capital y la riqueza en el conjunto de la sociedad. Una orientación en este sentido la da su referencia a desarrollos teóricos de la obra de Marx en el coloquio que convocó a diferentes investigadores para celebrar el centenario de su muerte. En él, Fals Borda destacó al marxista indú Barun De, quien planteaba que:

según la experiencia de la India la lucha de clases no podía verse como se ha visto tradicionalmente, esto es, como un conflicto de oposición directa entre contrarios, en una especie de relación binaria de oposición: la clase burguesa y la clase proletaria. Que en la práctica no existe esa lucha binaria sino una lucha múltiple de clases. Son diferentes clases que hacen alianzas o que suman sus recursos frente a diversos enemigos
[...] La posibilidad de una lucha multiclasista (Fals Borda, 1983, p. 15).

Lo cual no quiere decir que renuncie a tener en cuenta los postulados marxistas. Su valoración de la "vigencia de sus análisis del capitalismo", Fals Borda, 2003, p. 81) como el reconocimiento de sus aportes en los procesos revolucionarios y del desarrollo de la humanidad así lo testimonian.

Si quisiéramos encontrar a quién o a qué se orientarían las acciones que emprendan quienes luchan por el socialismo raizal, no habría dudas para establecer que Fals Borda lo ubica en el capitalismo del cual explícitamente afirma que "está llevando al mundo a la destrucción". Conclusión que deriva del análisis de los impactos del capitalismo actual bajo su forma neoliberal. Quizá pudiera encontrarse en el conjunto de su obra que si los Estados y sociedades de América Latina se hubieran orientado por la aplicación de aquellos principios de la Democracia Liberal que le fueron clásicos, hubiera podido esperar que una subversión moral produjera, de una manera más "natural", los cambios necesarios para orientarse hacia el socialismo. Todo su trabajo en la lucha por dar una redefinición territorial a la nación conducente a la constitución de una república regional unitaria, así lo indica. Es decir, su confianza en que desde la acción y el espíritu de los pueblos en aldeas y villorrios se produciría una fuerza que podría conducir a la transformación de los esquemas de dominación y de explotación. Sin embargo, el estudio de la historia nacional y mundial y el conocimiento de otras investigaciones antropológicas, religiosas, etc., y su seguimiento a procesos que se precipitaron en el mundo desde la década del 80 del siglo anterior con el neoliberalismo, le dieron elementos suficientes para concluir que la estrategia debía enfocarse hacia el desmoronamiento del capitalismo. Al mismo tiempo, la experiencia de las grandes movilizaciones 
a nivel mundial de los movimientos sociales en contra del neoliberalismo, le indicaron un camino para su transformación.

La dinámica de los movimientos sociales en América Latina le dio argumentos de una mayor contundencia sobre las posibilidades de que, para lograrlo, la violencia sólo sería necesaria en casos extremos. La misma experiencia de Lula con el Partido de los Trabajadores del Brasil fue reconocida con elogios en esta perspectiva. Igualmente, las que vivieron Argentina y Uruguay; sin que en ninguno de los tres países, sus gobernantes plantearan una orientación hacia el socialismo. Aunque, en ellos no fue estrictamente tan contundente la acción de los movimientos sociales; sin desconocer las grandes movilizaciones uruguayas en contra de algunas privatizaciones ni las del pueblo argentino cuando la crisis financiera lo condujo al momento más crítico de su historia.

Quizá, la mayor esperanza la ubicó en los procesos que viven Bolivia y Ecuador, en tanto que allí fue explícito el papel de los movimientos sociales en las transformaciones institucionales y constitucionales que se produjeron. Y no de cualquier manera, pues se realizaron en los dos países con más alto porcentaje de población indígena en Suramérica, con las implicaciones que ello tiene, en relación con sus tradiciones culturales, y su permanencia a través de las luchas de resistencia, en la construcción de la nueva sociedad. En cuanto tal, el socialismo raizal adquiría cuerpo porque se están transformando "las estructuras gubernamentales existentes", propiciando el camino hacia la construcción de una sociedad que garantice condiciones de dignidad y de justicia de su pueblo.

De allí que, reiterando, no podría derivarse del pensamiento de Fals Borda que su socialismo conduzca a la socialización de los principales medios de producción. Si bien para garantizar la "Dignidad y la justicia" se requiere una redistribución de los ingresos y de la propiedad, no necesariamente ello pasa por el traslado de la propiedad al Estado. Nuevamente estaríamos en frente de un punto de distanciamiento con los postulados marxistas. Pues, si bien argumenta que los pueblos requieren pasar del "reino de la necesidad al de la libertad", como lo planteaba Marx, no necesariamente ello se lograría con la limitación a toda iniciativa privada. Sería necesario, sí, garantizar una justicia social que posibilitara una forma de distribución de la riqueza y de acceso de la gran mayoría de la población, tanto a los bienes necesarios para su existencia material y biológica, como a todos aquellos bienes culturales y espirituales que les está vedado dentro del capitalismo.

Su definición de cultura podría ser de gran ayuda para clarificar la orientación que propone. Sobre todo si la entendemos como realizada dentro de esa nueva construcción social. Pues la define como:

elemento básico de las paz social, económica y política, como resultado del equilibrio entre razón y naturaleza que debe resolver la falsa dicotomía entre cuerpo y alma. Así una nueva "escala de valores". debe aparecer ante la crisis capitalista mundial, que redefina al trabajo "no como remisión ni rescate ni esclavitud", sino simplemente como "la realización de una vida plena y digna, en lo que ésta tiene de más gozo, intenso y cierto: la creación y el servicio" (Fals Borda, 2007, p. 142).

De lo cual se desprende que sólo podría efectuarse este postulado si se supera una sociedad que se sitúa en el mercado y en la cosificación de las relaciones humanas. Pues, sólo así el trabajo adquiriría esa connotación de desarrollo de las capacidades creativas de los seres humanos. Sería igualmente el en- 
cuentro con los espacios posibles de realización humana que los mismos seres humanos irían creando sin las limitaciones que hasta ahora le impone la voracidad del capital. Tendencia que muy bien podría coincidir en gran parte con los postulados ontológicos de Marx en los Manuscritos en cuanto que sólo en la sociedad comunista se podría efectuar una completa realización humana, y con los de Estanislao Zuleta al plantear que la capacidad de goce y creatividad humanas están limitados en una sociedad en que el ser humano se define por su relación o apropiación con las cosas; es decir, por la propiedad privada y las mercancías (Zuleta, 2004).

Por ello, la participación popular; es decir, la democracia radical, adquiere una importancia sin igual. Puesto que no estaría de acuerdo con que la historia repitiera el ejercicio de prácticas burocráticas en nombre del socialismo, como las de los estados socialistas de Europa del Este, que viciarían su realización. Tampoco la de la implementación de doctrinas externas a la vida de las comunidades y de los pueblos que distorsionaran lo que los pueblos, las comunidades y la ciudadanía han podido construir. Además, es necesario:

apelar a la fuente primigenia del poder que es el pueblo soberano. [...] Se necesita reconocer, articular y hacer respetar la organización propia o autónoma de la sociedad civil que se expresa en movimientos sociales y en espacios vitales mínimos, empezando con la gente de carne y hueso que habita veredas, laderas, barrancos, o valles, el "pueblito viejo" desarmado que vive de su fuerza de trabajo (Fals Borda, 2000, p. 47).

Su confianza en que allí es donde se origina la fuerza de la nueva sociedad, es la que da sentido a la recuperación y reconocimiento de sus prácticas culturales que den cuerpo a las demandas de la transformación social.

Son muchos otros los aspectos que podríamos considerar en relación con el proyecto de socialismo que Fals Borda presenta. Entre ellos, por ejemplo, todo lo relacionado con el territorio y sus implicaciones en la formación de un nuevo país y una nueva sociedad; o el significado de la tradición en la afirmación de los valores culturales. O hasta dónde llega la deuda con la cultura europea en relación con este campo. Sin embargo, para concluir, no podríamos dejar de mencionar dos aspectos que para él se convierten en imprescindibles para garantizar una nueva subversión moral: la I(A)P y la educación, en cuanto son parte constitutiva de cualquier proceso de transformación social. No en el significado que le asignaba la Ilustración europea, sino en relación con que ellas se articulan en la identificación y valoración de "las diversidades culturales, étnicas y de género" y en "cuanto desarraiga por las bases aquello que es congruente con las utopías" (Fals Borda, 2007, p. 102). No en relación con establecer otro parámetro o paradigma inamovible como lo impuso el logocentrismo europeo; más bien con el desarrollo de prácticas científicas que se colocan explícitamente a favor de las clases explotadas y oprimidas de la sociedad o de procesos de transformación social, hacia la formación de un nuevo humanismo no homogenizador sino situado en la diversidad. De igual manera, una educación y una pedagogía que no se establezcan por fuera de dichos procesos sino construidos a través de ellos, y, desde allí, contribuyan con sus desarrollos a la renovación de los saberes, desde las propias condiciones de vida cultural y social de las comunidades y pueblos.

De esta manera Fals Borda abre caminos de alternativa frente a la sociedad capitalista que desde su construcción, por las leyes mismas 
de su propia dinámica, ha generado desigualdad económica y social. A la vez, deja reflexiones que cuestionan al educador y al investigador respecto de la función social que como tales debe tener. Optando, sin dudarlo, por el compromiso de todos de coadyuvar en la realización de la "subversión moral" que sociedades como la nuestra requieren con urgencia. Por eso podríamos concluir con una cita de su texto de clausura del Congreso Mundial de Convergencia en Investigación Participativa 97: Estado del arte:

parece que aún podemos sobrevivir como humanos y controlar los desastres que autogeneramos; que todavía tenemos la posibilidad de reorganizarnos en otras formas más eficaces y satisfactorias, y de llegar a ser felices en alguna medida aceptable, con un nuevo humanismo (Fals Borda, 1998, p. 255).

\section{Referencias}

Fals Borda, O. et ál. (1962). La Violencia en Colombia. Bogotá: Tercer Mundo.

Fals Borda, O. et ál. (1972). El Reformismo por dentro en América Latina. Bogotá: Siglo Veintiuno.

Fals Borda, O. et ál. (1979). Campesinos de los Andes. Bogotá: Punta de Lanza.

Fals Borda, O. et ál. (1981). Las Revoluciones Inconclusas en América Latina 18091968. Bogotá: Siglo Veintiuno.

Fals Borda, O. et ál. (1982). El Socialismo que queremos: un nuevo pacto social y politico en Colombia. Cali: FUNCOP.

Fals Borda, O. et ál. (1983). Marx y el tercer mundo. En Fals Borda, O et ál. El Mar- xismo en Colombia. Bogotá: Universidad Nacional de Colombia.

Fals Borda, O. et ál. (1988). La insurgencia de las provincias: hacia un nuevo ordenamiento territorial para Colombia. Bogotá: Siglo Veintiuno.

Fals Borda, O. et ál. (1992). Vigencia de utopías en América Latina. Ponencia presentada en el VI Encuentro de Ciencias Sociales, Feria Internacional del Libro, Guadalajara, México. (Material fotocopiado).

Fals Borda, O. et ál. (1988). Compilación y análisis. "Participación popular: retos del futuro". Bogotá: ICFES, IEPRI, COLCIENCIAS.

Fals Borda, O. et ál. (2000). Acción y espacio: autonomía en la Nueva República. Bogotá: Tercer Mundo Editores en Coedición con la Universidad Nacional.

Fals Borda, O. et ál. (2001). Kaziyadu: Registro del reciente despertar territorial en Colombia. Bogotá: Ediciones Desde Abajo.

Fals Borda, O. et ál. (2003). Ante la crisis del país: Ideas-Acción. Bogotá: Ediciones El Áncora y Panamericana.

Fals Borda, O. et ál. (2007). Hacia el Socialismo Raizal y otros escritos. Bogotá: Ediciones Cepa y Desde abajo.

Fals Borda, O. et ál. (2008). La subversión en Colombia, el cambio social en la historia. Bogotá: Ediciones Fica-Cepa.

Jaramillo Salgado, D. (1997). Las huellas del Socialismo. Los discursos socialistas en Colombia 1919-1929. Coedición Universidad Autónoma del Estado de México, Toluca y Universidad del Cauca. Popayán: Toluca. 
Vargas Martínes, G. (2007). Colombia 1854: Melo, los artesanos y el socialismo. (La dictadura democrático-artesanal de 1854, expresión del socialismo utópico en Colombia). Medellín: La Oveja Negra.
Zuleta, E. (1986). Arte y Filosofía. Medellín: Percepción. 